
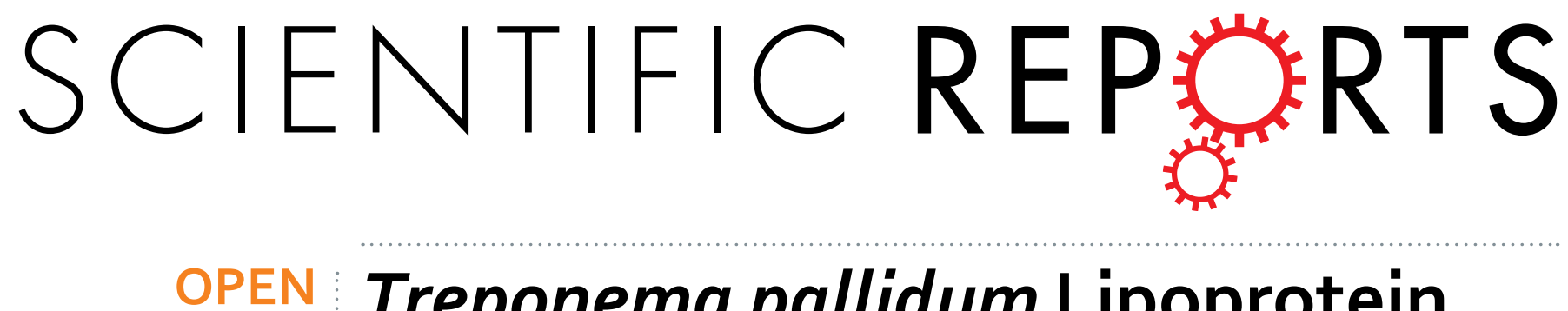

\title{
Treponema pallidum Lipoprotein TP0435 Expressed in Borrelia burgdorferi Produces Multiple Surface/Periplasmic Isoforms and mediates Adherence
}

Received: 28 January 2016

Accepted: 18 April 2016

Published: 10 May 2016

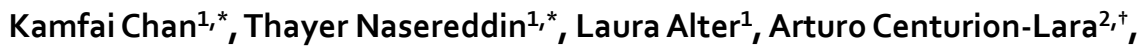 \\ Lorenzo Giacani² \& Nikhat Parveen ${ }^{1}$
}

The ability of Treponema pallidum, the syphilis spirochete to colonize various tissues requires the presence of surface-exposed adhesins that have been difficult to identify due to the inability to culture and genetically manipulate $T$. pallidum. Using a Borrelia burgdorferi-based heterologous system and gain-in-function approach, we show for the first time that a highly immunogenic lipoprotein TP0435 can be differentially processed into multiple isoforms with one variant stochastically displayed on the spirochete surface. TP0435 was previously believed to be exclusively located in T. pallidum periplasm. Furthermore, non-adherent $B$. burgdorferi strain expressing TP0435 acquires the ability to bind to a variety of host cells including placental cells and exhibits slow opsonophagocytosis in vitro similar to poor ex vivo phagocytosis of T. pallidum by host macrophages reported previously. This phenomenon of production of both surface and periplasmic immunogenic lipoprotein isoforms has possible implications in immune evasion of the obligate pathogen T. pallidum during infection.

Syphilis is a chronic, systemic, sexually transmitted disease that still affects millions of people worldwide. Syphilis is caused by the spirochete Treponema pallidum subspecies pallidum (T. pallidum), which was first isolated in 1912. Vertical transmission of this pathogen from mother to foetus is a leading cause of stillbirths in developing countries $^{1,2}$. The molecular mechanisms of syphilis pathogenesis still remain poorly understood mostly due to inability to culture and genetically manipulate T. pallidum. Identification of T. pallidum lipoproteins as adhesins s $^{3,4}$ like those in other pathogenic spirochetes ${ }^{5-8}$ implies their surface localization ${ }^{9}$ and was instrumental in challenging the assertion that lipoproteins of T. pallidum reside exclusively in the periplasmic space and are not surface-exposed ${ }^{3,10-20}$. Weak labelling of the highly immunogenic T. pallidum lipoproteins by sera from patients and experimentally infected animals on the surface of these spirochetes ${ }^{21,22}$, and observation of low density of integral transmembrane protein complexes by freeze-fracture electron microscopy on outer membrane (OM) of T. pallidum ${ }^{23,24}$; however, support significant periplasmic location of the lipoproteins.

Heterologous expression systems using related bacterial species have been previously used to identify and functionally characterize virulence factors of various pathogens including adhesins, such as the ActA and Internalin proteins of Listeria monocytogenes and Invasin of Yersinia pseudotuberculosis ${ }^{25-27}$. Lyme disease-causing Borrelia burgdorferi and T. pallidum are structurally and physiologically related spirochetal pathogens that express different lipoproteins. Even though the stealth pathogen T. pallidum expresses fewer surface proteins, B. burgdorferi is a useful surrogate to express T. pallidum proteins, determine their sub cellular location, and investigate their maturation and function because; (a) lipoproteins are processed through similar biochemical pathways ${ }^{28}$; (b) $46 \%$ of T. pallidum open reading frames have orthologs in B. burgdorferi with potentially overlapping functions ${ }^{29}$;

${ }^{1}$ Department of Microbiology, Biochemistry and Molecular Genetics, Rutgers-New Jersey Medical School, Newark, NJ 07103, USA. ${ }^{2}$ Department of Medicine, University of Washington, Seattle, WA 98104, USA. 'Present address: School of Public Health, Universidad Peruana Cayetano Heredia, Lima 30, Peru. *These authors contributed equally to this work. Correspondence and requests for materials should be addressed to N.P. (email: Parveeni@njms.rutgers. edu) 
(c) both have characteristic spiral shape, possess flagella in the periplasmic space (endoflagella), and lack lipopolysaccharide (LPS); and (d) are extracellular pathogens that cause systemic diseases.

Some genomic and physiological differences exist between T. pallidum and B. burgdorferi. B. burgdorferi can be grown in complex medium in vitro and genetically manipulated ${ }^{30-32}$ and its genome $(1.52 \mathrm{Mb})$ consists of a linear chromosome and numerous endogenous linear and circular plasmids ${ }^{33}$ encoding the majority of $\sim 132$ lipoproteins responsible for survival and colonization of tick vector and various hosts. In contrast, T. pallidum possesses a circular chromosome $(1.13 \mathrm{Mb})$ and no plasmids ${ }^{29}$ and expresses only 22 putative lipoproteins with mostly still unconfirmed localization. Long-term in vitro cultivation of infectious B. burgdorferi strains results in the loss of its endogenous plasmids rendering the spirochetes non-adherent to host cell lines and non-infectious in the mouse model. We used two high passage, poorly adherent, non-infectious B. burgdorferi strains (B314 and $\mathrm{B} 31 \mathrm{HP})$, which have lost different endogenous plasmids ${ }^{34}$ to investigate the role of highly expressed and immunogenic TP0435. B314 has more severe loss of adhesins-encoding plasmids, such as Lp54 (Supplementary Fig. S1).

Early in our studies, we determined the structural similarities to predict potential roles of two major lipoproteins, TP0171 and TP0435 using Phyre 2 site and M4T ${ }^{35}$. TP0435 showed structural homology with the new lipoprotein E (NlpE) of Escherichia coli, a known adhesin. We selected TP0435 (also known as the $17 \mathrm{kD}$ lipoprotein or Tpp17) for expression and to determine function of T. pallidum lipoprotein(s) using B. burgdorferi because of our interest in studying adherence mechanism of spirochetes. We show here that TP0435 is stochastically expressed on the surface of both B. burgdorferi and T. pallidum and this lipoprotein facilitates binding of the spirochetes to mammalian epithelial, glioma and placental cell lines.

\section{Results}

TP0435 is recognized by secondary syphilis patient serum on B. burgdorferi surface by Indirect Fluorescent Antibody (IFA) test. We cloned the tp0435 gene along with its upstream 500 nucleotides containing putative promoter region in a B. burgdorferi shuttle vector, which also possesses a codon-optimized firefly luciferase gene ${ }^{36}$, and then used the construct to transform B. burgdorferi strains and denoted them as B314(pTP) and $\mathrm{B} 31 \mathrm{HP}(\mathrm{pTP})$. B314 and B31HP strains transformed by vector alone designated as $\mathrm{B} 314(\mathrm{~V})$ and $\mathrm{B} 31 \mathrm{HP}(\mathrm{V})$, respectively were also generated as control strains.

IFA was conducted to assess whether antibodies in a syphilis patient serum detect TP0435 on intact B. burgdorferi. Surface labelling of both B. burgdorferi strains B314(pTP) and B31HP(pTP) and not control empty vector containing $\mathrm{B} 314(\mathrm{~V})$ and $\mathrm{B} 31 \mathrm{HP}(\mathrm{V})$ strains (Fig. 1 and Supplementary Fig. S2) indicate that the patient serum recognizes TP0435 but not the surface proteins of B. burgdorferi strains used in this study. TP0435 expression, processing, and transport across the bacterial cytoplasmic membrane here validates $B$. burgdorferi as a useful surrogate system for T. pallidum lipoproteins. The absence of flagella staining without permeabilization indicates that the spirochetes remained intact during IFA (Fig. 1a, c bottom, and Supplementary Fig. S2a,c bottom Panels). Interestingly, permeabilization of $\mathrm{B} 314(\mathrm{~V})$ and $\mathrm{B} 31 \mathrm{HP}(\mathrm{V})$ resulted in weak staining with secondary syphilis patient serum indicating some cross-reactivity with periplasmic protein(s). More intense staining of both TP0435 and periplasmic flagellin on permeabilization indicated that the majority of TP0435 is located in the periplasmic compartment of the spirochetes (Fig. 1d and Supplementary Fig. S2d). Indeed, on probing with syphilis patient serum, average fluorescence intensity did not change for $\mathrm{B} 314(\mathrm{~V})$ and $\mathrm{B} 31 \mathrm{HP}(\mathrm{V})$ control strains after permeabilization, while it increased to 2-3 fold in B314(pTP) and B31HP(pTP) after permeabilization (Supplementary Table S1).

TP0435 of T. pallidum produces multiple protein isoforms in B. burgdorferi. Using a mouse antiserum that we raised against purified recombinant TP0435 for immunoblotting of whole B. burgdorferi B314(pTP) cells lysate, we detected multiple TP0435 isoforms ranging between $14-17 \mathrm{kD}$ in size and are numbered starting with number 1 on the top, the largest protein band. The presence of protein isoforms is an unusual feature for bacterial exported proteins (Fig. 2a). Two protein gels were run and blotted in the same setup in duplicate and probed with three mouse antisera raised against spirochete proteins. Only weak cross reactivity of antibodies against TP0435 to control B314(V) strain indicates that the antiserum is specific for this protein. Limited Proteinase K treatment of intact live B314(V) and B314(pTP) strains indicated that OspC, a $21 \mathrm{kD}$ surface lipoprotein of $B$. burgdorferi that is expressed at high levels in B314 strain, was digested but not the periplasmic flagellar protein FlaB, confirming that only surface proteins are susceptible to this treatment. Furthermore, susceptibility of at least the largest isoform (band 1), and potentially also band 3 to proteolysis indicates that TP0435 is exposed on the bacterial surface. Similar results were obtained when the B31HP(pTP) strain was treated in an identical manner (Fig. 2b) indicating that multiple isoforms are not an artefact of expression of TP0435 in a particular B. burgdorferi strain. Multiple bands equivalent to isoforms in spirochetes were not detected with purified recombinant TP0435 as detected by Coomassie stain or Western blotting (Fig. 2a, right panels). It is currently believed by several researchers that the outer membrane of T. pallidum is deficient in transmembrane proteins ${ }^{23}$ and lipoproteins are anchored towards the periplasmic leaflet of spirochete outer membrane. However, association of TP0435 with membrane fraction was confirmed by phase partitioning with non-ionic detergent, triton X-114 ${ }^{37}$. Therefore, to determine whether different isoforms of TP0 435 are also associated with the outer membrane of $B$. burgdorferi or may be anchored to its inner membrane or peptidoglycan layer in the periplasmic compartment, we purified outer membrane vesicles (OMV) from B31HP(pTP). Observation of purified OMV by Differential Interference Contrast (DIC, Fig. 2c left) or after staining with our anti-B. burgdorferi OMV antibodies followed by anti-mouse antibodies conjugated to Alexa fluor 488 (Fig. 2c right) indicated the complete absence of intact/ghost spirochetes in the preparation. SDS-PAGE resolution followed by Western blotting on loading total proteins from more than $10^{8}$ spirochetes proteins showed the presence of an additional $14 \mathrm{kD}$ TP0 435 band and at least top two isoforms association with the OMV with only a barely visible band 3 (Fig. 2d); however, we cannot rule out the possibility that due to low yield of highly purified OMV, the lack of lower band association with outer membrane is only due 
a
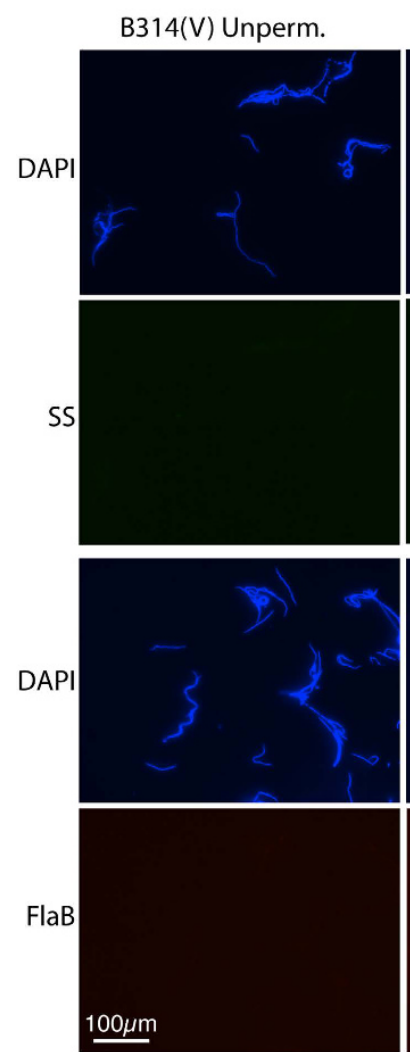

b

B314(V) Perm.
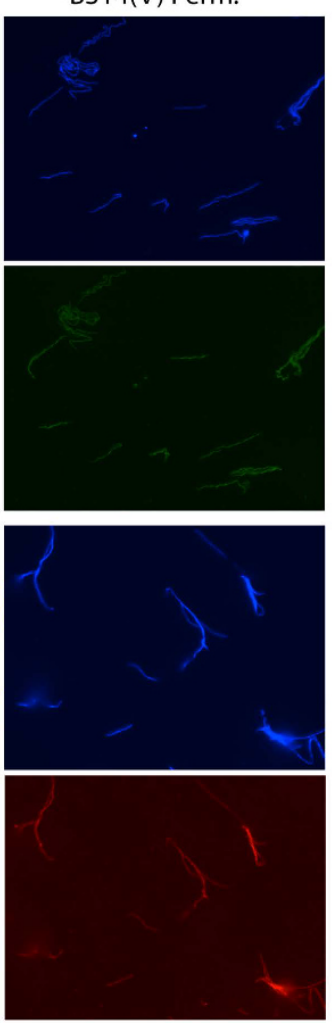

C B314(pTP) Unperm.
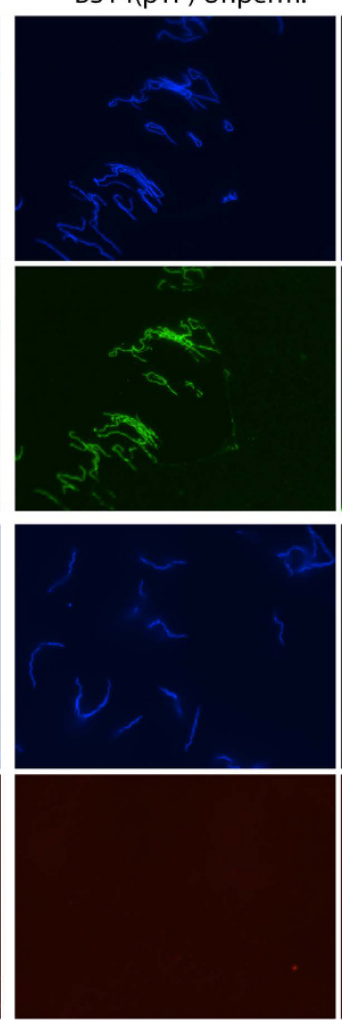

d

B314(pTP) Perm.
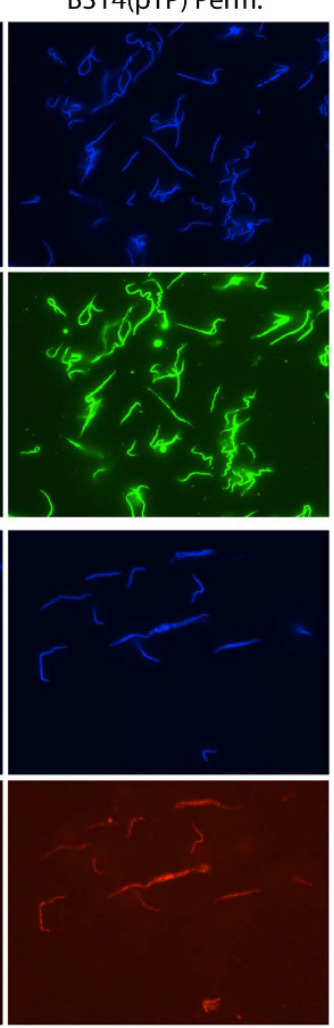

Figure 1. TP0435 is expressed on B. burgdorferi surface. (a) Absence of labelling of surface proteins of empty vector containing B314(V) control strain by secondary syphilis (SS) patient serum followed by treatment with anti-human Alexa fluor 488 conjugated secondary antibodies in IFA indicates that antibodies in this patient serum do not recognize B. burgdorferi surface proteins. All spirochetes in the respective fields were imaged after simultaneous staining with DAPI. Integrity of these bacteria during IFA was confirmed by lack of staining of periplasmic flagella with monoclonal antibodies against FlaB followed by reaction with anti-mouse TRITC conjugated antibodies. (b) Poor, albeit detectable, staining of B314(V) with SS patient serum on permeabilization indicates recognition of some periplasmic proteins of $B$. burgdorferi by this serum. (c) Punctate staining of majority of B. burgdorferi strain B314(pTP) with SS serum in IFA indicates the presence of TP0435 on spirochetes surface. Lack of periplasmic flagella staining again indicates that the integrity of outer membrane was maintained during IFA in this strain too. (d) Permeabilization of B314(pTP) results in more intense staining of TP0435 (Supplementary Table S1) indicating a significant presence of this protein also in the periplasmic region. Permeabilization also allowed intense staining of periplasmic flagella. Scale represents all panels in the figure.

to sensitivity of detection. Detection of OspC and not periplasmic flagellin further confirmed purity of our OMV preparation.

TP0435 is displayed on B. burgdorferi surface in a stochastic manner. Since the pattern of expression of TP0435 is similar in both B. burgdorferi strains, further studies were primarily conducted using B314 strain. No gold labelling was observed on the B. burgdorferi control B314(V) strain probed with the same secondary syphilis patient serum by Immunogold-Scanning Electron Microscopy (Immuno-SEM) (Fig. 3a,b) even in back-scattered images (BSI) of the same field (Fig. 3d,e) confirming that antibodies in this serum do not recognize surface proteins of B314 strain. Interestingly, only a few B. burgdorferi cells in each field showed TP0435 scattered on their surface, as detected by the presence of bound gold particles (Fig. 3c). Gold particles are more clearly visible in the BSI of the same field of view of B314(pTP) (Fig. 3f). Stronger gold labelling was observed in the inner concave face of the outer membrane accessed through accidental tearing of the OM (Fig. 4a) than on the outer convex surface of the OMV observed in the same field. Again, stochastic labelling with the gold particles is more clearly observed by BSI (Fig. 4b).

To determine if antibodies raised in mice against recombinant TP0435, which lacked the first 25 amino acids of translated protein, can recognize the conformational epitopes on B. burgdorferi surface or periplasmic region, we conducted IFA (Supplementary Fig. S3). Anti-TP0435 serum showed high background fluorescence probably due to haemolysis of blood collected to recover serum, making detection of the surface protein on B. burgdorferi difficult. Punctate TP0434 could be detected by IFA following permeabilization. 
a

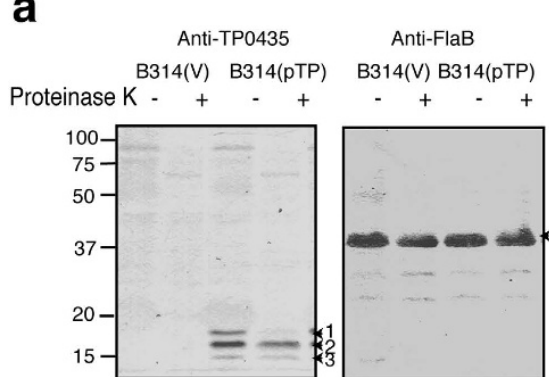

C

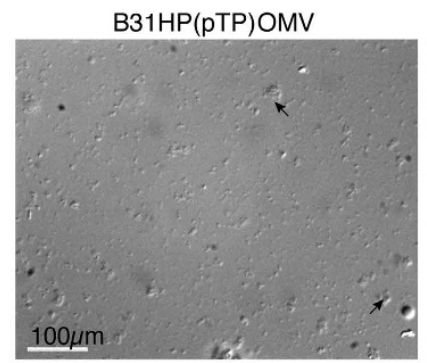

B31HP(pTP)OMV $\alpha \mathrm{OMV}+\alpha \mathrm{M}-\mathrm{AL}$ exa 488

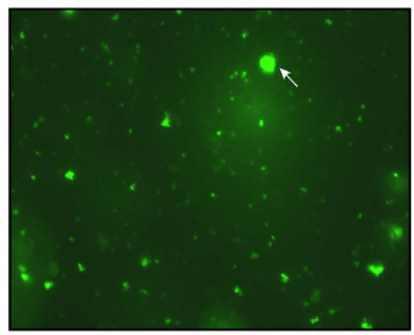

b

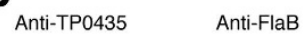

B31HP4(V) B31HP(pTP)B31HP(V) B31HP(pTP)

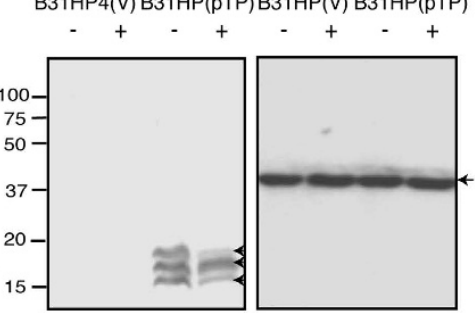

d

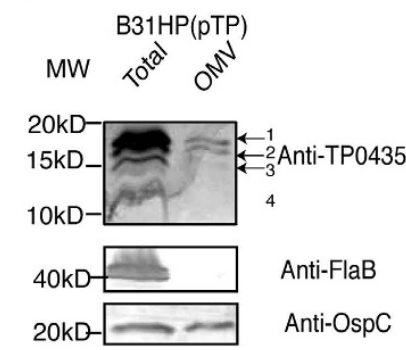

Figure 2. Expression of TP0435 lipoprotein of T. pallidum in B. burgdorferi produces multiple isoforms. (a) Analyses of total proteins of B314(V) and B314(pTP) with and without limited Proteinase K treatment were resolved by two SDS-PAGE gels with the same amount of respective samples loaded in duplicate in each gel. Western blotting of the gels using mouse anti-TP0435 serum showed the presence of multiple protein bands only when TP0435 is expressed. Limited Proteinase K treatment of intact B. burgdorferi cleaved at least the top TP0435 protein band number 1 (arrow) indicating that this isoform at minimum is surface exposed. Cleavage of protein band 3 likely also occurred to some extent. Negligible background staining indicates significant specificity of the mouse antibodies. Detection of intact FlaB (control for periplasmic proteins) and cleavage of the OspC (surface Borrelia lipoprotein) in the parallel blots confirms that only surface proteins are digested by limited Proteinase K treatment here. Arrows indicate FlaB and OspC protein monomers while higher oligomer of $\mathrm{OspC}$ is also observed. Clean preparation of recombinant TP0435 detected by Coomassie staining (Coom.) and Western blotting with anti-TP0435 serum (West.) with no bands equivalent to the isoforms seen in spirochetes preparation observed. (b) Results in 'a' were reproduced with $\mathrm{B} 31 \mathrm{HP}(\mathrm{pTP})$ and $\mathrm{B} 31 \mathrm{HP}(\mathrm{V})$ strains. (c) Clean Outer membrane Vesicles (OMV) preparation of $\mathrm{B} 31 \mathrm{HP}(\mathrm{pTP})$ without contamination with spirochetal structures was obtained, as detected by Differential Interference Contrast (DIC, left) or by staining with anti-B. burgdorferi OMV mouse serum followed by anti-mouse $(\alpha-\mathrm{M})$ secondary antibodies conjugated to Alexa fluor 488 (right). Arrows indicate cluster of OMVs. (d) OspC of B. burgdorferi and three isoforms (bands 1, 2, and probably 3) of TP0435 remain associated with the OMV as detected by Western blotting. Absence of flagellin in OMV preparation confirms that the preparation is not contaminated with major periplasmic proteins. Lane marked as 'Total' indicates that the spirochetes total proteins were loaded in the lane.

Stochastic distribution of TP0435 is also observed on T. pallidum surface. Western blot analysis of total proteins of T. pallidum Nichols strain resolved by SDS-PAGE using mouse anti-TP0435 antibodies consistently showed the presence of at least two bands even though only the smallest protein is predominantly detected (Fig. 5a). TP0435 protein isoforms of different sizes in B. burgdorferi suggests differential processing of translated protein; however, the presence of isoforms in T. pallidum could not be ascertained. The level of $\sim 14 \mathrm{kD}$ TP0435 in $B . b u$ rgdorferi is quite low indicating that some differences exist in the level of processed membrane proteins in two spirochetes possibly reflecting differences conferred by in vitro and in vivo growth conditions for B. burgdorferi and T. pallidum, respectively. In our experiments, the top $\sim 17 \mathrm{kD}$ protein band was detected in T. pallidum only when protein extract from more spirochetes $\left(1-2 \times 10^{8}\right.$ per lane) was loaded in gel.

Antibodies present in secondary syphilis patient sera react with a variety of T. pallidum antigens. Therefore, to test whether TP0435 is indeed also present on the T. pallidum surface, immuno-SEM of T. pallidum was conducted using anti-TP0435 mouse serum. T. pallidum treated with mouse normal prebled serum did not show any attached gold particles (Fig. 5c). Surface labelling of only some T. pallidum spirochetes in each field of view was observed with anti-TP0435 mouse serum (Fig. 5d) and was similar to our immuno-SEM results with the B314(pTP) strain of B. burgdorferi when treated with secondary syphilis patient serum (Figs $3 \mathrm{~b}$ and $4 \mathrm{a}$ ). Labelling of TP0435 increased for T. pallidum harvests in which the outer membrane of spirochetes recovered from the rabbit testes was disrupted and accessibility of antibodies increased to the abundant TP0435 periplasmic isoforms (Supplementary Fig. S4a,b). Together these results suggest that B. burgdorferi and T. pallidum can both process newly translated TP0435 proteins to produce mature variants that are differentially localized either exclusively to the periplasmic space or to both the periplasm and the surface of the spirochetes. Surface display of TP0435 in each spirochete appears random (Figs 3c,4a and 5d) indicating that TP0435 post-translational processing in each bacterial cell present in a population occurs independently and stochastically. 

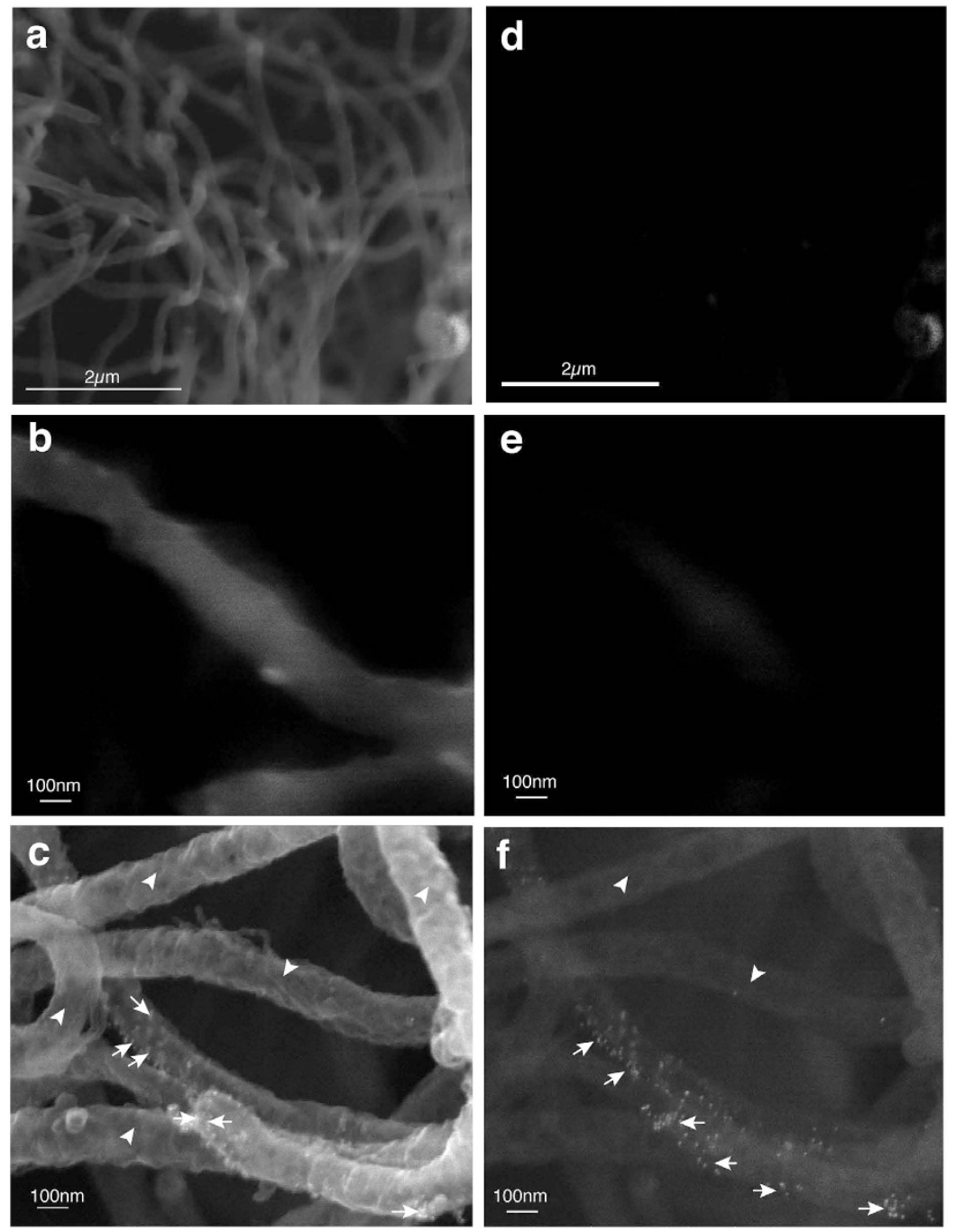

Figure 3. Immuno-SEM shows TP0435 is displayed on B. burgdorferi cells surface in a stochastic manner. (a,b) Absence of gold particles attached to B314(V) control spirochetes probed with secondary syphilis (SS) patient serum followed by anti-human gold conjugated antibodies (at $25 \mathrm{x}$ and 100x magnifications) confirms that this serum does not recognize surface proteins of B. burgdorferi strain B314. (c) Immuno-SEM imaging of B314(pTP) probed with SS serum at high magnification (100x) showed attached gold particles only on some of the B. burgdorferi cells (arrows). Arrowheads indicate the spirochetes without any gold particles attached. (d,e) Absence of attached gold particles by Back Scattered Imaging (BSI) of B314(V) in the field shown in the Fig. 3a,b, respectively confirms that the patient serum does not recognize surface proteins of B314 strain. (f) Stochastic expression of TP0435 on B314(pTP) surface in Fig. 3c is more clearly visible in BSI of the same field.

Post-translational processing likely produces multiple TP0435 isoforms. We were unable to sequence TP0435 immuno-enriched from B. burgdorferi strain and resolved by SDS-PAGE by Edman degradation suggesting that the $\mathrm{NH}_{2}$-terminal end of TP0435 is blocked. Suggested localization of the protein isoforms in the outer membrane versus inner membrane by LipoP1.0 program falls between 30-70\%. Liquid Chromatography and Tandem Mass Spectrometric (LC-MS/MS) analyses of TP0435 after enrichment from T. pallidum and B31HP by immunoaffinity chromatography detected different peptides of TP0435 after chymotrypsin digestion (Fig. 5b and Supplementary Fig. S5a); however, did not recognize the N-terminal modified/ unmodified peptides. Identification of peptides, modified and unmodified by MS analyses depends on known sequence and mass. Differential processing at different Cysteine residues followed by lipidation could be responsible for multiple TP0435 isoforms production (Supplementary Fig. S5a,b). Interestingly, unlike B. burgdorferi, several T. pallidum predicted lipoproteins show multiple cysteines presence within the first 50 amino acids of translated Open Reading Frames (Supplementary Table S2). Since the nature of the lipid moiety attached to the $\mathrm{NH}_{2}$-terminal cysteine in TP0435 and its mass is not known, we were unable to determine the sequence of $\mathrm{NH}_{2}$-terminal lipidated peptides in the isoforms by MS analyses of either T. pallidum or B31HP(pTP) despite trying four different MS core facilities of well-known universities in the USA. Furthermore, it is difficult to obtain milligrams of purified proteins from spirochetes required for identification of lipidated peptides. 

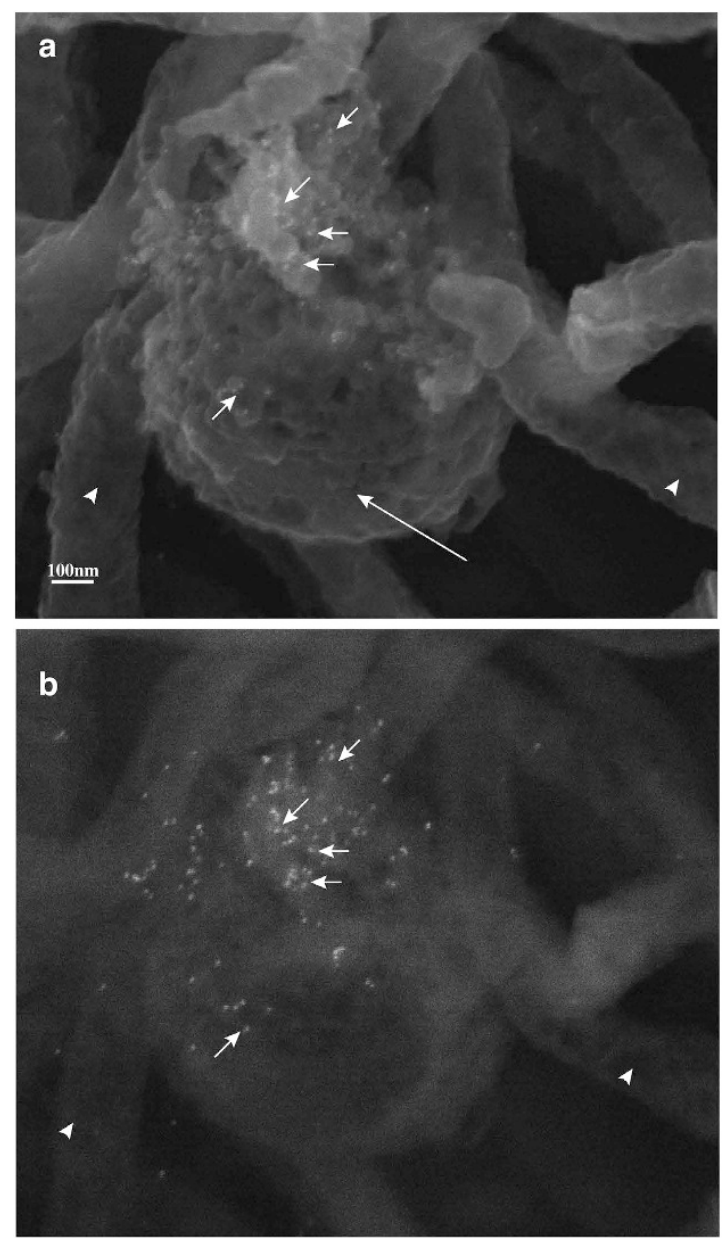

Figure 4. TP0435 is more prominently present in the inner, periplasmic face of the outer membrane (OM). (a) Inner concave face of accidently ripped OM of one B. burgdorferi shows more attached gold particles compared with those on the outer surface of another bacterium indicating that the most of TP0435 is present in periplasmic region during actively growing bacteria. Round structure indicated by a larger arrow indicates the convex outer surface of an OMV. (b) Attached gold particles in the field shown in 'a' are more clearly observed by the Back Scattered Imaging (BSI).

TP0435 is an adhesin that facilitates binding to the mammalian cells. The next logical step was to determine the role of the surface localized TP0435 in spirochete pathogenesis using a tissue culture system. To conduct these studies, the structural homology of TP0435 protein with E. coli NlpE (new lipoprotein E) protein was used as foundation because the crystal structure of NlpE and its function were already determined ${ }^{38}$. Using BLAST, NlpE shows $35 \%$ of sequence identity and $48 \%$ similarity with TP0435. Additionally, Phyre 2 program predicted TP0435 structural similarity with NlpE at $99.97 \%$ confidence. The predicted structure closely resembles the monomer TP0435 crystal structure determined very recently ${ }^{39}$ encouraging us to assess if TP0435 has a role in adherence.

To determine whether TP0435 is also an adhesin, we examined the ability of B314(pTP) strain to bind to embryonic human kidney epithelial 293 and placental BeWo cell lines. Bioluminescence measurement of B. burgdorferi B314 expressing TP0435 showed a statistically significant, 50-70-fold increase in binding to these two cell lines as compared to the B314(V) control strain (Fig. 6a,b), confirming TP0435 involvement in attachment to human cells. Because firefly luciferase activity is also sensitive to the $\mathrm{pH}$ of the medium and available ATP levels ${ }^{40}$, we also used a complementary technique to determine binding using ${ }^{35} \mathrm{~S}$ methionine-cysteine labelled $B$. burgdorferi strains (Fig. 6c). Again, B314(pTP) showed a statistically significant higher level of binding to both 293 and C6 Glioma cell lines as compared to the control B314(V) strain. Unfortunately, BeWo cells did not bind to the NUNC break apart plates used for these assays affecting integrity of the monolayers during washing and thus, binding of labelled B. burgdorferi (data not shown). However, our gain-of-function results determined by two complementary techniques with 293 cells included in both assays indicate that TP0435 present on the spirochete surface indeed functions as an adhesin (Fig. 6a,c).

Slow opsonophagocytosis of $B$. burgdorferi strains expressing TP0435 similar to that observed in T. pallidum. B. burgdorferi strains surrogate system also helped us detect phagocytosis-mediated killing by mouse macrophage cell line J774A.1. After incubation of B314(V), B314(pTP), B31HP(V) and B31HP(pTP) with 
a b
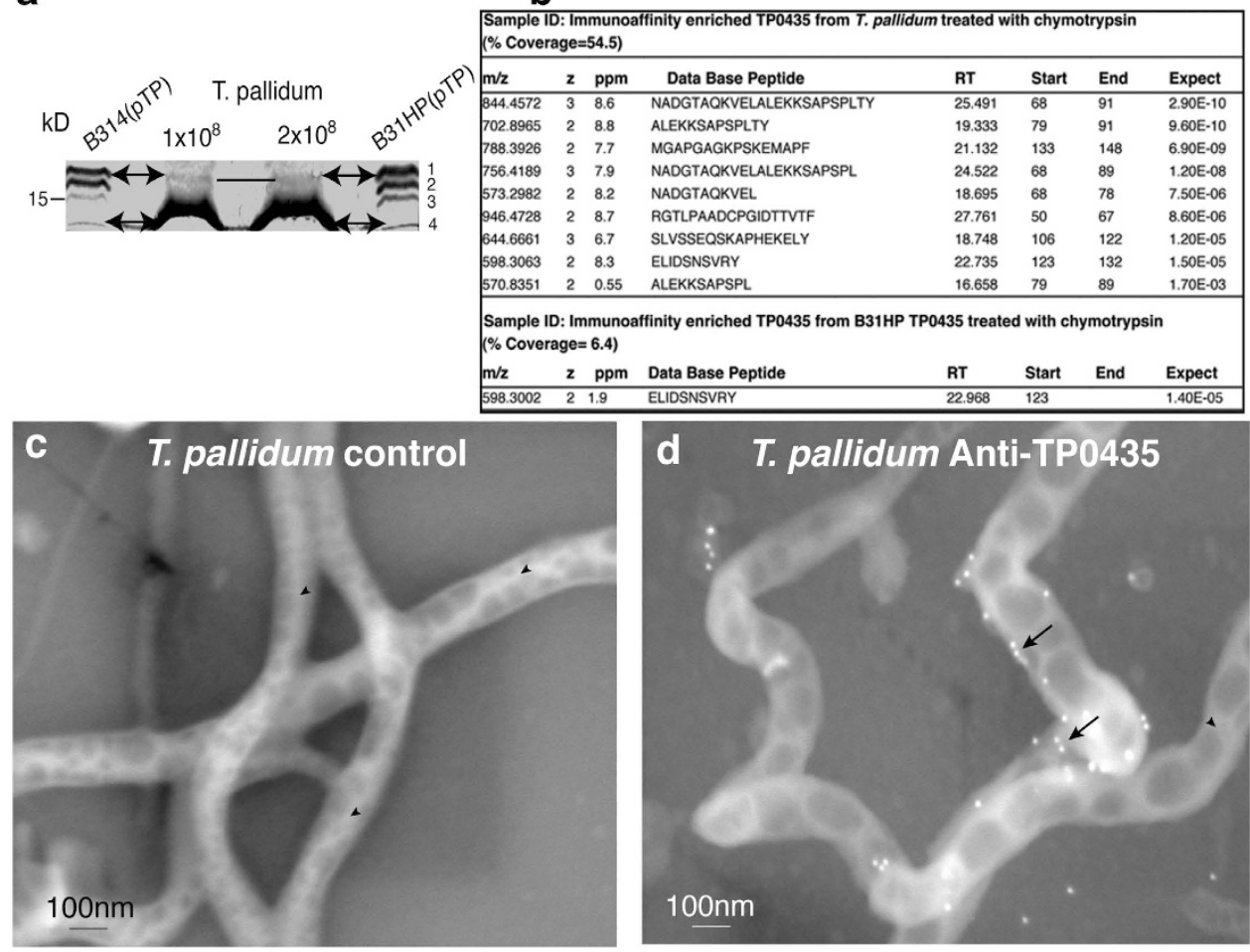

Figure 5. Random pattern of TP0435 surface expression is also observed in T. pallidum cells. (a) Protein bands likely equivalent to at least two isoforms $(17 \mathrm{kD}$ and $14 \mathrm{kD})$ detected in B314 $(\mathrm{pTP})$ and B31HP(pTP) strains are also discernible in T. pallidum by Western blotting. (b) Liquid Chromatography-tandem MassSpectrometric (MS/MS) analyses of TP0435 in T. pallidum Nichols strain and B31HP(pTP) after chymotrypsin digestion (Supplementary Fig. S5a). Table shows the derived peptide sequence, start and end of the peptide, parts per million $(\mathrm{ppm}), \mathrm{m} / \mathrm{z}$ representing mass $(\mathrm{m})$ divided by charge number $(\mathrm{z})$, retention time of the peptide in column (RT), and the best expected value the peptide (Expect). (c) Lack of attached gold particles on T. pallidum surface when probed with normal mouse serum followed by anti-mouse gold antibodies (arrowheads) shows that the prebled mouse serum or secondary antibodies do not non-specifically recognize T. pallidum proteins. (d) T. pallidum probed with anti-TP0435 mouse antibodies followed by secondary antibodies conjugated to gold particles indicate surface localization of TP0435 occur randomly in some T. pallidum cells (arrows).

the specific mouse antibodies, spirochetes were added to the macrophage cells, incubated at $37^{\circ} \mathrm{C}$, fixed, stained and examined microscopically (Fig. 7). Extracellular spirochetes are green and were stained by respective antibodies prior to permeabilization while red fluorescence was obtained due to post-permeabilization staining and indicates phagocytosed spirochetes. In our negative control, J774A.1 macrophage cell line failed to phagocytise $\mathrm{B} 314(\mathrm{pTP})$ and $\mathrm{B} 31 \mathrm{HP}(\mathrm{pTP})$ strains preincubated with antibodies against periplasmic FlaB protein even after $6 \mathrm{~h}$ of co-incubation of bacteria with J774A.1 cells (Fig. 7a,b). Phagocytosis occurred within $2 \mathrm{~h}$ of co-incubation of macrophage cell line and $\mathrm{B} 314(\mathrm{pTP})$ and $\mathrm{B} 31 \mathrm{HP}(\mathrm{pTP})$ spirochetes preincubated with antibodies against B. burgdorferi surface protein OspC (Fig. 7c,d) indicating normal phagocytosis rate when antibodies against surface $B$. burgdorferi protein are used for opsonization. Bright red phagocytised and often degraded spirochetes were detected by staining after permeabilization of cells after $6 \mathrm{~h}$ co-incubation of J774A.1 cells with B314(pTP) or B31HP(pTP), which were opsonized with anti-TP0435 antibodies (Fig. 7e,f). Both of these spirochete strains also lost bioluminescence significantly when incubated with J774A.1 for $4 \mathrm{~h}$ indicating killing after phagocytosis (data not shown). These results suggest that $B$. burgdorferi cells expressing TP0435 were phagocytised and ultimately degraded even though opsonophagocytosis occurred rather slowly. Control B. burgdorferi strains with empty vector, $\mathrm{B} 314(\mathrm{~V})$ and $\mathrm{B} 31 \mathrm{HP}(\mathrm{V})$, preincubated with anti-TP0435 serum remained extracellular (green) even after $6 \mathrm{~h}$ of co-incubation with macrophage cell line (Supplementary Fig. S6). Our results with non-infectious B. burgdorferi here also agree with previous findings with $T$. pallidum where opsonophagocytosis occurs at a slow rate compared to that observed for other pathogens likely due to limited surface exposure of these proteins ${ }^{41}$.

\section{Discussion}

Receptor-mediated adherence to host cells plays a critical role in the life of extracellular pathogens that can persist in the host for long durations despite stimulation of a strong adaptive immune response. T. pallidum long-term survival in the host, which can lead to the serious clinical manifestations of tertiary syphilis is suggested to be facilitated by the uncommon paucity of proteins on the surface of this spirochete ${ }^{3,10-20}$. However, this scenario does not address how T. pallidum colonizes various human tissues during syphilis. 
a

\begin{tabular}{|c|c|c|c|c|c|c|c|}
\hline $\begin{array}{l}V \text { pTP } \\
\text { BeWo }\end{array}$ & $\begin{array}{c}\text { B314- } \\
\text { V pTP } \\
293\end{array}$ & $\underset{\mathrm{NC}}{\mathrm{V} \text { pTP }}$ & $\begin{array}{l}\text { Color Bar } \\
\text { Min. } 42182 \\
\text { Max. } 1.0065 \mathrm{e}+06 \\
-1.0\end{array}$ & Quantificati & $\mathrm{n}$ of binding by biolum & nescence $\mathrm{m}$ & asuremen \\
\hline & & & $=0.8$ & Cell Lines & B. burgdorferi strains & $\begin{array}{l}\text { Average } \\
\text { Binding }\end{array}$ & SD \\
\hline & & & & BeWo & B314(V) & $1.34 \times 10^{4}$ & $2.44 \times 10^{3}$ \\
\hline & & & $=0.6$ & BeWo & B314(pTP) & $6.27 \times 10^{5 * *}$ & $1.05 \times 10^{5}$ \\
\hline & & & & 293 & B314(V) & $1.28 \times 10^{4}$ & $1.50 \times 10^{3}$ \\
\hline & & & $=0.4$ & 293 & B314(pTP) & $9.05 \times 10^{5 * \star}$ & $9.76 \times 10^{4}$ \\
\hline & & & -0.2 & No Cell (NC) & B314(V) & $1.26 \times 10^{4}$ & $1.84 \times 10^{3}$ \\
\hline & & & & No Cell (NC) & $\mathrm{B} 314(\mathrm{pTP})$ & $8.41 \times 10^{4}$ & $1.17 \times 10^{4}$ \\
\hline
\end{tabular}

C

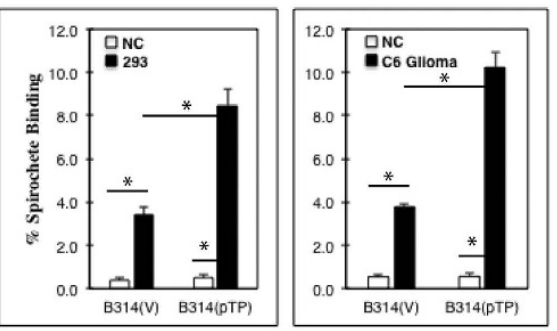

Figure 6. TP0435 is an adhesin that facilitates binding to epithelial, Glial and placental cells. (a) B314(pTP) strain is adherent to BeWo and HEK 293 cells with 50- and 70-fold increase in binding as compared to the control B314(V) strain to these cells as detected by measurement of light emitted by the attached luminescent spirochetes. NC indicates 'No Cell' control. (b) Adherence levels depicted in 'a' are quantified. Attachment of $\mathrm{B} 314(\mathrm{pTP})$ is significantly higher than $\mathrm{B} 314(\mathrm{~V})$ binding to the respective cell lines as determined by student $\mathrm{T}$ test $\left({ }^{* *} \mathrm{p}<0.001\right)$. Binding of $\mathrm{B} 314(\mathrm{~V})$ to both cell lines is not significantly different from $\mathrm{B} 314(\mathrm{pTP})$ strain binding to NC control. (c) Binding of ${ }^{35}$ S-labeled B314(pTP) strain to HEK 293 and rat C6 Glioma cells increased in statistically significant manner as compared to $\mathrm{B} 314(\mathrm{~V})$ binding $\left({ }^{*} \mathrm{P}<0.001\right.$ by student $\mathrm{T}$ test). Binding of $\mathrm{B} 314(\mathrm{~V})$ to both cells was also significantly higher $\left({ }^{*} \mathrm{P}<0.001\right)$ as compared to binding of this strain to NC control.

\section{Opsonophagocytosis J774A.1 Cell line}
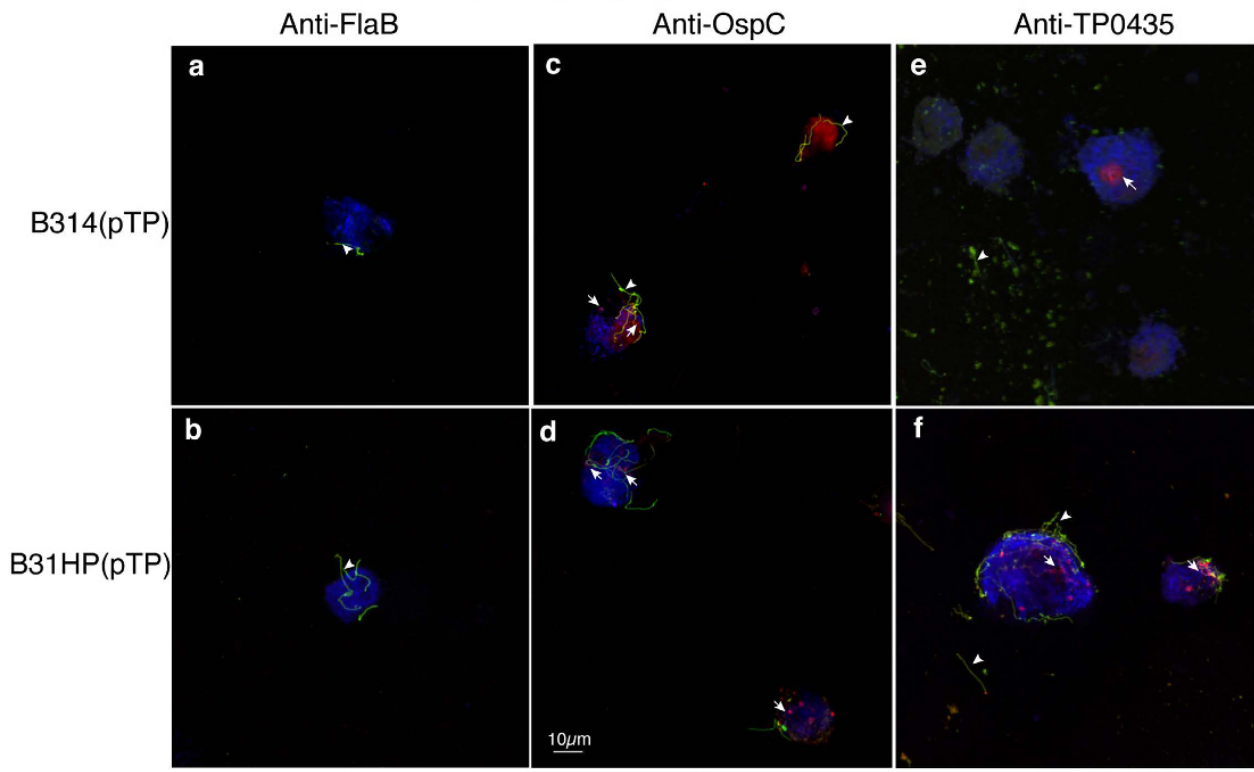

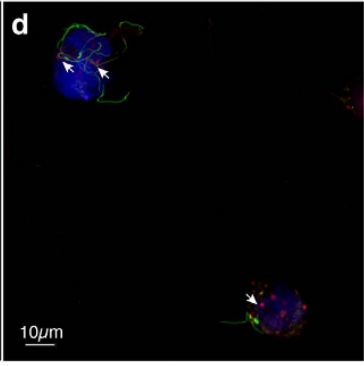

$2 \mathrm{~h}$

$6 \mathrm{~h}$

Figure 7. Anti-TP0435 mouse antibodies facilitate only low opsonophagocytosis of B314(pTP) and B31HP(pTP) strains. (a,b) J774A.1 mouse macrophage cell line failed to phagocytise B31HP(pTP) and $\mathrm{B} 314$ (pTP) preincubated with antibodies against periplasmic protein FlaB even after $6 \mathrm{~h}$ of co-incubation. Extracellular spirochetes (green/yellow marked by arrowhead) were observed on staining of B. burgdorferi with anti-B. burgdorferi OMV antibodies followed by Alexa fluor 488 conjugated anti-mouse antibodies before permeabilization, while to detect intracellular spirochetes, after permeabilization counterstaining was done with anti-OMV antibodies followed by anti-mouse antibodies conjugated to TRITC. (c,d) Preincubation of $\mathrm{B} 314(\mathrm{pTP})$ and $\mathrm{B} 31 \mathrm{HP}(\mathrm{pTP})$ with antibodies against B. burgdorferi surface protein OspC showed phagocytosis by J774A. 1 cells within $2 \mathrm{~h}$ of co-incubation shown as red intracellular degrading spirochetes. $(\mathbf{e}, \mathbf{f}) \mathrm{B} 31 \mathrm{HP}(\mathrm{pTP})$ and B314(pTP) preincubated with anti-TP0435 antibodies bind to J774A.1 mouse macrophage cell line. After $6 \mathrm{~h}$ of co-incubation, both bound or unbound extracellular B. burgdorferi (green marked by arrowhead) and intracellular spirochetes (red marked by arrow) are observed indicating slow but detectable opsonophagocytosis. Scale represents all panels in the figure. 
Our studies support that $B$. burgdorferi is a suitable heterologous system to functionally characterize T. pallidum proteins and possible virulence factors. Detection of multiple TP0435 isoforms in B. burgdorferi was unexpected and may allow escape of spirochetes from immune response. Different protein isoforms originating from the same precursor by differential post-translational processing and modification are not common in bacteria. Indeed, Lpp of a laboratory strain of commensal E. coli is the only other known bacterial lipoprotein that possesses two isoforms ${ }^{42,43}$, one located in the periplasmic space with a C-terminal lysine anchored to the peptidoglycan layer (bound form), while the second form is integrally associated with the outer membrane and is surface-exposed, even though its transmembrane domain(s) is yet to be identified ${ }^{42}$. $\mathrm{NH}_{2}$-terminal of both forms was suggested to be acylated, indicating that both versions are lipoproteins. Interestingly, C-terminal lysine residue is also present in TP0435 (Supplementary Fig. S5a); however, the experimental evidence for the exact anchor for periplasmic TP0435 is not yet available.

OMV preparations of B. burgdorferi expressing TP0435 show the presence of multiple isoforms associated with outer membrane. Top surface band $(\sim 17 \mathrm{kD}$, band number 1$)$ appears to represent less than one third of the total TP0435 present in B. burgdorferi (Fig. 2a,b). This observation strongly suggests that a majority of TP0435 is periplasmic with only a smaller fraction being surface exposed. Low abundance of surface TP0435 protein in T. pallidum, which could be equivalent to the isoform 1 of TP0435 present in B. burgdorferi, was detected only after overloading the gel (Fig. 5a). A caveat of comparing the levels of each isoform present in the spirochetes by Western blotting is that antibodies against a specific epitope present only in a particular isoform may be missing and thus, could diminish detection of this isoform. For example, limited Proteinase $\mathrm{K}$ treatment suggests that band 3 of TP0435 may also be displayed on the surface to some extent but its quantity, as detected by Western analysis, appears to be low (Fig. 2). Even then, our findings confer with previous suggestion by other researchers that a significant amount of T. pallidum proteins are present in periplasmic region of the spirochetes recovered from rabbit testes ${ }^{11,23}$. A possible explanation is that a majority of the free spirochetes recovered from rabbit testes are those unable to bind to the host tissues and possess only a small amount of this lipoprotein displayed on the outer membrane surface ${ }^{44}$. Supporting this premise, we observed that TP0435 is stochastically expressed on the surface of only some T. pallidum cells (Fig. 5c). Based upon three adjacent or overlapping lipoboxes in TP0435, we postulate that differential cleavage of this surface immunogenic exported protein followed by lipidation could be a critical and unique mechanism for T. pallidum to produce lipoprotein isoforms. Only a limited amount of the outer membrane associated TP0435 then results in exposure on bacterial surface (Figs 2,3,4 and 5). We suggest that a limited surface exposure versus complete periplasmic presence of TP0435 in different T. pallidum cells during infection may ultimately lead to alternative pathogen fates.

Adherence through NlpE protein activates the CpxR/CpxA two component system and has been implicated in suppressing the extracytoplasmic toxicities triggered by mutant or misfolded outer membrane protein(s) in E. colit ${ }^{45}$. This suppression is achieved by up regulation of the production of the periplasmic protease, DegP, which degrades defective proteins ${ }^{46}$. Protein turnover in the periplasmic space could also be important in T. pallidum, considering that most of its lipoproteins are suggested to be present in this subsurface compartment. TP0435 is likely a bifunctional protein, similar to that observed for many other spirochete surface proteins ${ }^{47-49}$, with different roles played by surface and periplasmic versions of TP0435. Previously, two roles proposed for periplasmic TP0435 were: (i) redox sensor; or, (ii) communication of its redox/oligomerization state to downstream effectors $^{39}$. We predict that immunogenic TP0435 location predominantly in the periplasmic space of the majority of disseminating T. pallidum cells particularly later in infection allows long-term sustenance of this obligate pathogen in the host, which is a necessary condition to develop late syphilis manifestations. Based upon our opsonophagocytosis results with B. burgdorferi and (Fig. 7) and slow phagocytosis of only a subpopulation of T. pallidum previously observed ${ }^{41,50}$, we postulate that the spirochetes that express TP0435 on their surface during the early stage of infection mediate adherence to various host cells. These T. pallidum cells are still protected in the blood stream since adaptive immune response is not yet established against its immunogenic proteins including TP0435. After development of a specific humoral response, T. pallidum cells that display TP0435 on their surface are negatively selected in the bloodstream, and only bacteria that lack TP0435 on their surface survive during dissemination. Thus, by the time an adaptive immune response against TP0435 is established, it is no longer needed on the surface of free T. pallidum while those still expressing TP0435 on the surface remain attached to the target cells, facilitating colonization of the specific tissues probably in the immunoprivileged sites.

\section{Methods}

Ethics Statement. New Zealand white rabbits were used for T. pallidum strain propagation. Animal care was provided in accordance with the Guide for the Care and Use of Laboratory Animals and procedures were conducted under protocols approved by the University of Washington Institutional Animal Care and Use Committee (IACUC) under protocol number 4243-01. Polyclonal antibodies against recombinant TP0435 were produced in $\mathrm{BALB} / \mathrm{c}$ mice using the previously described protocol ${ }^{1}$. All mouse experiments were performed in accordance with all provisions of the Animal Welfare Act, the Guide for the Care and Use of Laboratory Animals, and the PHS Policy on Humane Care and Use of Laboratory Animals. These experiments were conducted under the protocol number 14011D0617 approved by the Rutgers Biomedical and Health Sciences IACUC. Coded, secondary syphilis patient serum was provided for this work by Dr. Sheila Lukehart.

Cultivation of B. burgdorferi. High passage B. burgdorferi strains $\mathrm{B} 314$ and $\mathrm{B} 31 \mathrm{HP}^{34}$ were grown in BSKII medium containing $6 \%$ rabbit serum at $33^{\circ} \mathrm{C}$. 
Isolation and purification of Outer membrane Vesicles (OMV) of B31HP(pTP). OMV were separated from cytoplasmic cylinder using citrate buffer and purified by sucrose gradient centrifugation as described previously ${ }^{48,51}$.

Production of antibodies in mice against recombinant TP0435. Antibodies were raised against polyhistidine tagged recombinant purified TP0435 in BALB/c mice using our previously described proto$\mathrm{col}^{48}$. Detailed method used, with minor modifications in the previously described protocol, is provided in the Supplementary information.

T. pallidum strain propagation. T. pallidum Nichols $\operatorname{strain}^{52}$, originally provided by James N. Miller, University of California, Los Angeles, CA, was propagated in New Zealand white rabbits by means of intratesticular (IT) inoculation as previously reported ${ }^{53}$ and described in the supplementary information.

Mammalian cells culture. C6 (rat) glioma and human embryonic kidney 293 cell lines in our collection (originally obtained from Dr. John Leong's laboratory at Tufts University School of Medicine) were grown at $37^{\circ} \mathrm{C}$ and $5 \% \mathrm{CO}_{2}$ atmosphere as previously described ${ }^{54}$. BeWo cells (obtained from American Type Culture Collection) were cultured in F-12K medium supplemented with 10\% FBS and Penicillin/Streptomycin (P/S) mixture. Mouse macrophage J774A.1 cell line was grown in Dulbecco's Modified Eagle's Medium (DMEM) supplemented with 10\% FBS and P/S mixture, except antibiotic mixture was eliminated during the J774A.1 cells co-incubation with B. burgdorferi strains for phagocytosis experiment. DMEM, FBS and BSA were obtained from commercial sources.

Immunoaffinity enrichment of TP0435. After washing B. burgdorferi $\mathrm{B} 31 \mathrm{HP}(\mathrm{pTP})$ culture three times with the suspension buffer ( $50 \mathrm{mM}$ Tris- $\mathrm{HCl}$, pH7.4, $150 \mathrm{mM} \mathrm{NaCl}, 5 \mathrm{mM} \mathrm{EDTA}$ ), lysis was carried out with $1.7 \%$ TritonX100 containing suspension buffer i.e., lysis buffer, in the presence of protease inhibitor cocktail (Sigma P8849). After removing lysed bacterial pellet by centrifugation, proteins in the supernatant were precipitated with three volumes of cold acetone and after drying in air, pellet was resuspended in solubilization buffer, i.e., suspension buffer containing $0.2 \%$ Triton-X100 and protease inhibitor cocktail. Immunoaffinity enrichment of TP0435 from B. burgdorferi was conducted using a two-step process. In the first step, antibodies against B. burgdorferi cross-linked with protein $\mathrm{G}$ were used to remove majority of Borrelia proteins and supernatant containing significantly reduced level of $B$. burgdorferi proteins recovered after centrifugation. Protein $\mathrm{G}$ beads crosslinked to TP0435 mouse antiserum were then used to further enrich TP0435 protein from this supernatant. After washing, the beads boiled in protein loading dye were resolved by $12.5 \%$ SDS-PAGE and stained with Sypro Ruby (T. pallidum) or silver stain (B. burgdorferi). The first enrichment step was eliminated for immunoprecipitation and enrichment of TP0435 from T. pallidum. Gel segment containing enriched TP0435 from B. burgdorferi $(4-5 \mathrm{~mm})$ was sent to Tufts University Core facility to determine N-terminal sequence by Edman degradation method.

Protein Identification Using Reversed-phase Liquid Chromatography Electrospray Tandem Mass Spectrometry (LC-MS/MS). A 4-5 mm segment of gel region containing TP0435 was excised from the gel and subjected to in-gel digestion with high fidelity chymotrypsin. The peptides formed from the digested samples were analyzed by on-line LC-MS/MS technique. The LC separation was performed using a NanoAcquity UPLC system (Waters) on an Easy-Spray PepMap ${ }^{\circledR}$ column $(75 \mathrm{um} \times 15 \mathrm{~cm}$, Thermo Scientific) with a linear gradient from $2-30 \%$ B ( $0.1 \%$ formic acid in acetonitrile) followed by washing at $50 \%$ B at a flow rate of $600 \mathrm{nl} / \mathrm{min}$. The MS/MS analysis was performed using a LTQ Orbitrap Velos mass spectrometer (Thermo Scientific). After a survey scan, 6 most intense precursor ions were selected for subsequent fragmentation using HCD with normalized collision energy of 30. Both precursor and fragment ions were analyzed in the FT mode in the Orbitrap at mass resolution of 30000 and 7500 , respectively. The analytical peak lists were generated from the raw data using an, in-house software, PAVA $^{55}$. The MS/MS data were searched against the UniProt database using a University of California at San Francisco search engine, Protein Prospector, available at http://prospector.ucsf.edu/prospector/ mshome.htm.

IFA and Immuno-SEM of B. burgdorferi and T. pallidum. IFA of B. burgdorferi was conducted using previously reported protocol that is briefly described in the supplementary information. After centrifugation of B. burgdorferi grown to mid logarithmic phase at low speed $(4,000 \times \mathrm{g})$, the medium was discarded and culture fixed with 16\% paraformaldehyde solution (Electron Microscopy Sciences, Hatfield, PA) for 20 more minutes with light vortex every five minutes. Bacteria were washed twice with sodium phosphate buffer $(0.2 \mathrm{M}$ concentration, $\mathrm{pH} 7.2$ ) containing $0.2 \% \mathrm{BSA}$ (PBS/BSA). After incubation with the secondary syphilis patient serum overnight at $30^{\circ} \mathrm{C}$, culture was washed three times with PBS/BSA. Bacteria were incubated at $30^{\circ} \mathrm{C}$ with goat anti-human IgG antibody conjugated with $10 \mathrm{~nm}$ gold particles used at 1:3 dilutions (Abcam, MA) for $2 \mathrm{~h}$. After three washings with PBS/BSA, spirochetes were fixed in $2.5 \%$ glutaraldehyde for 30 minutes at room temperature and then dehydrated with a series of ethanol. After dehydration, culture pellets were dried in liquid $\mathrm{CO}_{2}$ (Tousimis Critical Point Dryer, Autosamdri-815, MD). Dried bacteria were then mounted onto aluminium SEM stubs with conductive carbon tape and then coated with $1.5 \mathrm{~nm}$ of carbon in a Denton Vacuum Evaporator 502B (Moorestown, NJ), and the specimens were examined and pictures taken by Gregory Hendricks at the University of Massachusetts Medical School, Core EM Facility using an FEI Quanta 200 FEG MK II scanning electron microscope.

Fresh T. pallidum cells were processed in similar manner except that the specific mouse polyclonal antiserum raised against TP0435 was used followed by a goat anti-mouse secondary antibody conjugated with $10 \mathrm{~nm}$ gold particles (Sigma-Aldrich, St. Louis, MO). Bacteria mounted on carbon SEM stubs (Electron Microscopy Sciences, 
Hatfield, PA) were coated with carbon in a Denton Vacuum Evaporator DV-502 (Moorestown, NJ) and images taken by Geoffrey Perumal at the Analytical Imaging Facility of Albert Einstein College of Medicine using a Zeiss Supra 40 Field Emission Scanning Electron Microscope (Carl Zeiss Microscopy, LLC, North America), with a backscatter detector using an accelerating voltage of $10 \mathrm{KV}$.

Binding assays. For determination of binding by bioluminescence detection, $B$. burgdorferi cells were counted and concentration adjusted to $\sim 3.7 \times 10^{7}$ cells $/ \mathrm{ml}$. Fifty microliter of culture suspension was added per well of a 96-well BD Falcon plate with black walls containing mammalian cell line monolayers. After centrifugation at $233 \times \mathrm{g}$ for 5 minutes, the plate was incubated at $37^{\circ} \mathrm{C}$ with $5 \% \mathrm{CO}_{2}$ for 1 hour to allow binding of bacterial cells. After three washes with $150 \mu \mathrm{l}$ PBS/BSA (five minutes) without shaking, $100 \mu \mathrm{l}$ of BSKII-RS containing $0.15 \mathrm{mg} / \mathrm{ml} \mathrm{D}$-luciferin was added to each well. Bioluminescence was measured using an IVIS-50 instrument (Perkin-Elmer, MA). Binding assays with radiolabel B. burgdorferi were carried out using previously described protocol $^{56}$.

Phagocytosis of $\boldsymbol{B}$. burgdorferi expressing TP0435. $\quad \mathrm{B} 314(\mathrm{pTP})$ and $\mathrm{B} 31 \mathrm{HP}(\mathrm{pTP})$ and controls, $\mathrm{B} 314(\mathrm{~V})$ and $\mathrm{B} 31 \mathrm{HP}(\mathrm{V})$, were incubated for 30 minutes with anti-TP0435 polyclonal mouse antibodies diluted 1:100 in J774A.1 medium. As positive and negative controls, B314(pTP) and B31HP(pTP) were preincubated with antibodies against surface and periplasmic B. burgdorferi proteins OspC and FlaB, respectively. After incubation of mouse macrophage J774A.1 cell line monolayers at $50 \%$ confluence with opsonised bacteria in medium without antibiotics for $2 \mathrm{~h}, 3 \mathrm{~h}$, and $6 \mathrm{~h}$ at $37^{\circ} \mathrm{C}$ in $5 \% \mathrm{CO}_{2}$ incubator (also $4 \mathrm{~h}$ for bioluminescence measurement only), co-incubated cells were fixed for $1 \mathrm{~h}$ using $3 \%$ paraformaldehyde in PBS. After fixation and blocking with PBS containing 5\% BSA and 5\% heat inactivated goat serum, samples were incubated with antibodies raised against crude OMV preparation of B. burgdorferi followed by secondary antibodies conjugated to Alexa fluor 488 to label extracellular spirochetes. We had already raised antibodies against crude OMV preparation from infectious $B$. burgdorferi strain in BALB/c mice using the protocol described previously ${ }^{48}$. J774A.1 cells were then stained with wheat agglutinin lectin conjugated with Alexa fluor 647. Cells were then permeabilized with cold methanol and B. burgdorferi counterstained with the anti-OMV primary antiserum followed by a secondary antiserum conjugated with TRITC to stain phagocytised bacteria.

\section{References}

1. Gerbase, A. C., Rowley, J. T., Heymann, D. H., Berkley, S. F. \& Piot, P. Global prevalence and incidence estimates of selected curable STDs. Sexually transmitted infections 74 Suppl 1, S12-16 (1998).

2. Goldenberg, R. L. \& Thompson, C. The infectious origins of stillbirth. Am J Obstet Gynecol 189, 861-873 (2003).

3. Brinkman, M. B. et al. A novel Treponema pallidum antigen, TP0136, is an outer membrane protein that binds human fibronectin. Infection and immunity 76, 1848-1857 (2008).

4. Cameron, C. E., Brouwer, N. L., Tisch, L. M. \& Kuroiwa, J. M. Defining the interaction of the Treponema pallidum adhesin Tp0751 with laminin. Infection and immunity 73, 7485-7494 (2005).

5. Cinco, M. New insights into the pathogenicity of leptospires: evasion of host defences. New Microbiol 33, 283-292 (2010).

6. Kovacs-Simon, A., Titball, R. W. \& Michell, S. L. Lipoproteins of bacterial pathogens. Infection and immunity 79, 548-561 (2011).

7. Hindersson, P. et al. Interaction of spirochetes with the host. Research in microbiology 143, 629-639 (1992).

8. Kenedy, M. R., Lenhart, T. R. \& Akins, D. R. The role of Borrelia burgdorferi outer surface proteins. FEMS Immunol Med Microbiol 66, 1-19 (2012).

9. Cameron, C. E. In Pathogenic Treponema Molecular and Cell Biology (eds Radolf, J. D. \& Lukehart, S. A.) Ch. 11, 237-266 (Caiser Aademic Press, 32 Hewitts Lane, Wymondham, Norfolk NR18 0JA, England, 2006).

10. Radolf, J. D., Norgard, M. V. \& Schulz, W. W. Outer membrane ultrastructure explains the limited antigenicity of virulent Treponema pallidum. Proc Natl Acad Sci USA 86, 2051-2055 (1989).

11. Cox, D. L., Chang, P., McDowall, A. W. \& Radolf, J. D. The outer membrane, not a coat of host proteins, limits antigenicity of virulent Treponema pallidum. Infection and Immunity 60, 1076-1083 (1992).

12. Bourell, K. W., Schulz, W., Norgard, M. V. \& Radolf, J. D. Treponema pallidum rare outer membrane proteins: analysis of mobility by freeze-fracture electron microscopy. J Bacteriol 176, 1598-1608 (1994).

13. Radolf, J. D. et al. Characterization of outer membranes isolated from Treponema pallidum, the syphilis spirochete. Infection and immunity 63, 4244-4252 (1995).

14. Blanco, D. R. et al. Recombinant Treponema pallidum rare outer membrane protein 1 (Tromp1) expressed in Escherichia coli has porin activity and surface antigenic exposure. J Bacteriol 178, 6685-6692 (1996).

15. Akins, D. R. et al. Tromp1, a putative rare outer membrane protein, is anchored by an uncleaved signal sequence to the Treponema pallidum cytoplasmic membrane. J Bacteriol 179, 5076-5086 (1997).

16. Cameron, C. E. et al. Opsonic potential, protective capacity, and sequence conservation of the Treponema pallidum subspecies pallidum Tp92. The Journal of infectious diseases 181, 1401-1413 (2000).

17. Hazlett, K. R. et al. TP0453, a concealed outer membrane protein of Treponema pallidum, enhances membrane permeability. $J$ Bacteriol 187, 6499-6508 (2005).

18. Tomson, F. L., Conley, P. G., Norgard, M. V. \& Hagman, K. E. Assessment of cell-surface exposure and vaccinogenic potentials of Treponema pallidum candidate outer membrane proteins. Microbes Infect 9, 1267-1275 (2007).

19. Izard, J. et al. Cryo-electron tomography elucidates the molecular architecture of Treponema pallidum, the syphilis spirochete. Journal of bacteriology 191, 7566-7580 (2009).

20. Anand, A. et al. Bipartite Topology of Treponema pallidum Repeat Proteins C/D and I: Outer membrane insertion, trimerization, and porin function requires A C-terminal beta-barrel domain. J Biol Chem 290, 12313-12331 (2015).

21. Salazar, J. C., Hazlett, K. R. \& Radolf, J. D. The immune response to infection with Treponema pallidum, the stealth pathogen. Microbes Infect 4, 1133-1140 (2002).

22. Schroder, N. W., Eckert, J., Stubs, G. \& Schumann, R. R. Immune responses induced by spirochetal outer membrane lipoproteins and glycolipids. Immunobiology 213, 329-340 (2008).

23. Radolf, J. D. Treponema pallidum and the quest for outer membrane proteins. [Review]. Molecular Microbiology 16, 1067-1073 (1995).

24. Blanco, D. R., Miller, J. N. \& Lovett, M. A. Surface antigens of the syphilis spirochete and their potential as virulence determinants. Emerging infectious diseases 3, 11-20 (1997). 
25. Isberg, R. R., Voorhis, D. L. \& Falkow, S. Identification of invasin: a protein that allows enteric bacteria to penetrate cultured mammalian cells. Cell 50, 769-778 (1987).

26. Kocks, C. et al. L. monocytogenes-Induced Actin Assembly requires the actA gene product, a surface protein. Cell 68, 521-532 (1992).

27. Mengaud, J., Ohayon, H., Gounon, P., Mege, R. M. \& Cossart, P. E-cadherin is the receptor for internalin, a surface protein required for entry of L. monocytogenes into epithelial cells. Cell 84, 923-932 (1996)

28. Setubal, J. C., Reis, M., Matsunaga, J. \& Haake, D. A. Lipoprotein computational prediction in spirochaetal genomes. Microbiology 152, 113-121 (2006).

29. Fraser, C. M. et al. Complete genome sequence of Treponema pallidum, the syphilis spirochete. Science 281, 375-388 (1998).

30. Samuels, D., Mach, K. \& Garon, C. Genetic transformation of the Lyme disease agent Borrelia burgdorferi with coumarin-resistant gyrB. J Bacteriol 176, 6045-6049 (1994).

31. Stewart, P. E., Thalken, R., Bono, J. L. \& Rosa, P. Isolation of a circular plasmid region sufficient for autonomous replication and transformation of infectious Borrelia burgdorferi. Mol Microbiol 39, 714-721. (2001).

32. Elias, A. F. et al. New antibiotic resistance cassettes sui for genetic studies in Borrelia burgdorferi. J Mol Microbiol Biotechnol 6, 29-40 (2003).

33. Fraser, C. M. et al. Genomic sequence of a Lyme disease spirochaete, Borrelia burgdorferi. Nature 390, 580-586 (1997).

34. Sadziene, A., Wilske, B., Ferdows, M. \& Barbour, A. The cryptic $\operatorname{ospC}$ gene of Borrelia burgdorferi B31 is located on a circular plasmid. Infect Immun 61, 2192-2195 (1993).

35. Fernandez-Fuentes, N., Madrid-Aliste, C. J., Rai, B. K., Fajardo, J. E. \& Fiser, A. M4T: a comparative protein structure modeling server. Nucleic Acids Res 35, W363-368 (2007).

36. Blevins, J. S., Revel, A. T., Smith, A. H., Bachlani, G. N. \& Norgard, M. V. Adaptation of a luciferase gene reporter and lac expression system to Borrelia burgdorferi. Appl Environ Microbiol 73, 1501-1513 (2007).

37. Radolf, J. D., Chamberlain, N. R., Clausell, A. \& Norgard, M. V. Identification and localization of integral membrane proteins of virulent Treponema pallidum subsp. pallidum by phase partitioning with the nonionic detergent triton X-114. Infect Immun 56, 490-498 (1988).

38. Hirano, Y., Hossain, M. M., Takeda, K., Tokuda, H. \& Miki, K. Purification, crystallization and preliminary X-ray crystallographic analysis of the outer membrane lipoprotein NlpE from Escherichia coli. Acta crystallographica. Section F, Structural biology and crystallization communications 62, 1227-1230 (2006).

39. Brautigam, C. A., Deka, R. K., Liu, W. Z. \& Norgard, M. V. Insights into the potential function and membrane organization of the TP0435 (Tp17) lipoprotein from Treponema pallidum derived from structural and biophysical analyses. Protein science: a publication of the Protein Society 24, 11-19 (2015).

40. Chan, K., Alter, L., Barthold, S. W. \& Parveen, N. Disruption of bbe02 by insertion of a luciferase gene increases transformation efficiency of Borrelia burgdorferi and allows live imaging in Lyme disease susceptible C3H mice PLos One 10 (6), (2015).

41. Marangoni, A. et al. Phagocytosis of Treponema pallidum and reactive oxygen species production by isolated rat Kupffer cells. Med Microbiol Immunol 192, 183-188 (2003).

42. Cowles, C. E., Li, Y., Semmelhack, M. F., Cristea, I. M. \& Silhavy, T. J. The free and bound forms of Lpp occupy distinct subcellular locations in Escherichia coli. Molecular microbiology 79, 1168-1181 (2011).

43. Cupp-Vickery, J. R., Peterson, J. C., Ta, D. T. \& Vickery, L. E. Crystal structure of the molecular chaperone HscA substrate binding domain complexed with the IscU recognition peptide ELPPVKIHC. Journal of molecular biology 342, 1265-1278 (2004).

44. Blanco, D. et al. Isolation of the outer membranes from Treponema pallidum and Treponema vincentii. J Bacteriol 176, 6088-6099 (1994).

45. Snyder, W. B., Davis, L. J., Danese, P. N., Cosma, C. L. \& Silhavy, T. J. Overproduction of NlpE, a new outer membrane lipoprotein, suppresses the toxicity of periplasmic LacZ by activation of the Cpx signal transduction pathway. Journal of bacteriology 177, 4216-4223 (1995).

46. Danese, P. N. \& Silhavy, T. J. The sigma(E) and the Cpx signal transduction systems control the synthesis of periplasmic proteinfolding enzymes in Escherichia coli. Genes \& development 11, 1183-1193 (1997).

47. Coburn, J. \& Cugini, C. Targeted mutation of the outer membrane protein P66 disrupts attachment of the Lyme disease agent, Borrelia burgdorferi, to integrin alphavbeta3. Proc Natl Acad Sci USA 100, 7301-7306 (2003).

48. Parveen, N. \& Leong, J. M. Identification of a candidate glycosaminoglycan-binding adhesin of the Lyme disease spirochete Borrelia burgdorferi. Molecular Microbiology 35, 1220-1234 (2000).

49. Skare, J. T. et al. The Oms66 (p66) protein is a Borrelia burgdorferi porin. Infect Immun 65, 3654-3661 (1997).

50. Lukehart, S. A., Shaffer, J. M. \& Baker-Zander, S. A. A subpopulation of Treponema pallidum is resistant to phagocytosis: possible mechanism of persistence. J Infect Dis 166, 1449-1453 (1992).

51. Skare, J. T. et al. Virulent strain associated outer membrane proteins of Borrelia burgdorferi. Journal of Clinical Investigation 96, 2380-2392 (1995).

52. Nichols, H. J. a. \& Hough, W. H. Demonstration of Spirochaeta pallida in the cerebrospinal fluid from a patient with nervous relapse following the use of Salvarsan. JAMA:The Journal of the American Medical Association 60, 108-110 (1913).

53. Lukehart, S. A. \& Marra, a. C. M. Isolation and laboratory maintenance of Treponema pallidum. Vol. 7, 12A.1.1-12A.1.18 (Wiley Online Library 2007).

54. Parveen, N., Robbins, D. \& Leong, J. M. Strain variation in glycosaminoglycan recognition influences cell-type-specific binding by Lyme disease spirochetes. Infection and Immunity 67, 1743-1749 (1999).

55. Guan, S., Price, J. C., Prusiner, S. B., Ghaemmaghami, S. \& Burlingame, A. L. A data processing pipeline for mammalian proteome dynamics studies using $s$ isotope metabolic labeling. Mol Cell Proteomics 10, M111 010728 (2011).

56. Magoun, L. et al. Variable small protein (Vsp)-dependent and Vsp-independent pathways for glycosaminoglycan recognition by relapsing fever spirochaetes. Mol Microbiol 36, 886-897 (2000).

\section{Acknowledgements}

We are thankful to Dr. Sheila Lukehart for providing genomic DNA of T. pallidum and a de-identified secondary syphilis patient serum for these studies. This work was funded by: National Institute of Allergy and Infectious Diseases grant RC1AI086027 to NP, the ASTDA Developmental Award to LG, and The International Collaborations in Infectious Disease Research (ICIDR) U01AI115497 award from NIH to NP, LG and ACL. The funders had no role in study design, data collection and analysis, decision to publish, or preparation of the manuscript. Scanning electron microscopy of B. burgdorferi was conducted at the University of Massachusetts Medical School Core EM Facility supported by the NIH through the shared instrument grant program S10RR021043, and of T. pallidum at Albert Einstein Medical Center Core EM facility. Luke Fritzky at Rutgers Confocal core facility captured Confocal Z-stack images of opsonophagocytosis using a Nikon-A1R confocal microscope equipped with a 60x Plan Apo VC oil immersion objective. The Bio-Organic Biomedical Mass Spectrometry Resource at UCSF (director, A.L. Burlingame) supported by Howard Hughes Medical Institute and 
the National Institute of General Medical Sciences grant no. P41GM103481 identified the peptides of TP0435 from T. pallidum and B31HP expressing TP0435. We are also grateful to Drs. Sheila Lukehart (University of Washington), Ronald Iorio (University of Massachusetts Medical School), and Purnima Bhanot (Rutgers New Jersey Medical School) for their critical review of the initial version of the manuscript.

\section{Author Contributions}

K.C., T.N., N.P. conducted most of the experiments; L.A. determined plasmid profile of B. burgdorferi strains and L.G. and A.C.-L. cultured T. pallidum in rabbit testes for Western blotting and Immuno-SEM; K.C. analysed data, N.P. and L.G. designed experiments and wrote the paper.

\section{Additional Information}

Supplementary information accompanies this paper at http://www.nature.com/srep

Competing financial interests: The authors declare no competing financial interests.

How to cite this article: Chan, K. et al. Treponema pallidum Lipoprotein TP0435 Expressed in Borrelia burgdorferi Produces Multiple Surface/Periplasmic Isoforms and mediates Adherence. Sci. Rep. 6, 25593; doi: 10.1038/srep25593 (2016).

cc) (i) This work is licensed under a Creative Commons Attribution 4.0 International License. The images or other third party material in this article are included in the article's Creative Commons license, unless indicated otherwise in the credit line; if the material is not included under the Creative Commons license, users will need to obtain permission from the license holder to reproduce the material. To view a copy of this license, visit http://creativecommons.org/licenses/by/4.0/ 